\title{
preparación mecánica de áridos
}

\author{
ALVARO SARMIENTO FARINÓS, ingeniero industrial
}

S I N O P S I S

La preparación de áridos para abastecer las obras que necesitan de ellos se ha centralizado, constituyendo centrales que, como las de hormigón, a las que con frecuencia acompañan, están formadas de una serie de órganos mecánicos, convenientemente concordantes unos con otros, de forma tal que su producción, características y continuidad, no solo resulte economicamente explo-

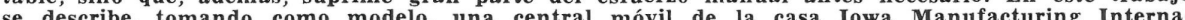

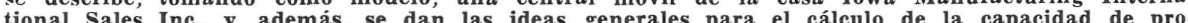
dución del modelo Pitmaster en función de la abertura de las quijadas de la machacadora, molino y curva granulométrica de la gravera que se ha de explotar.

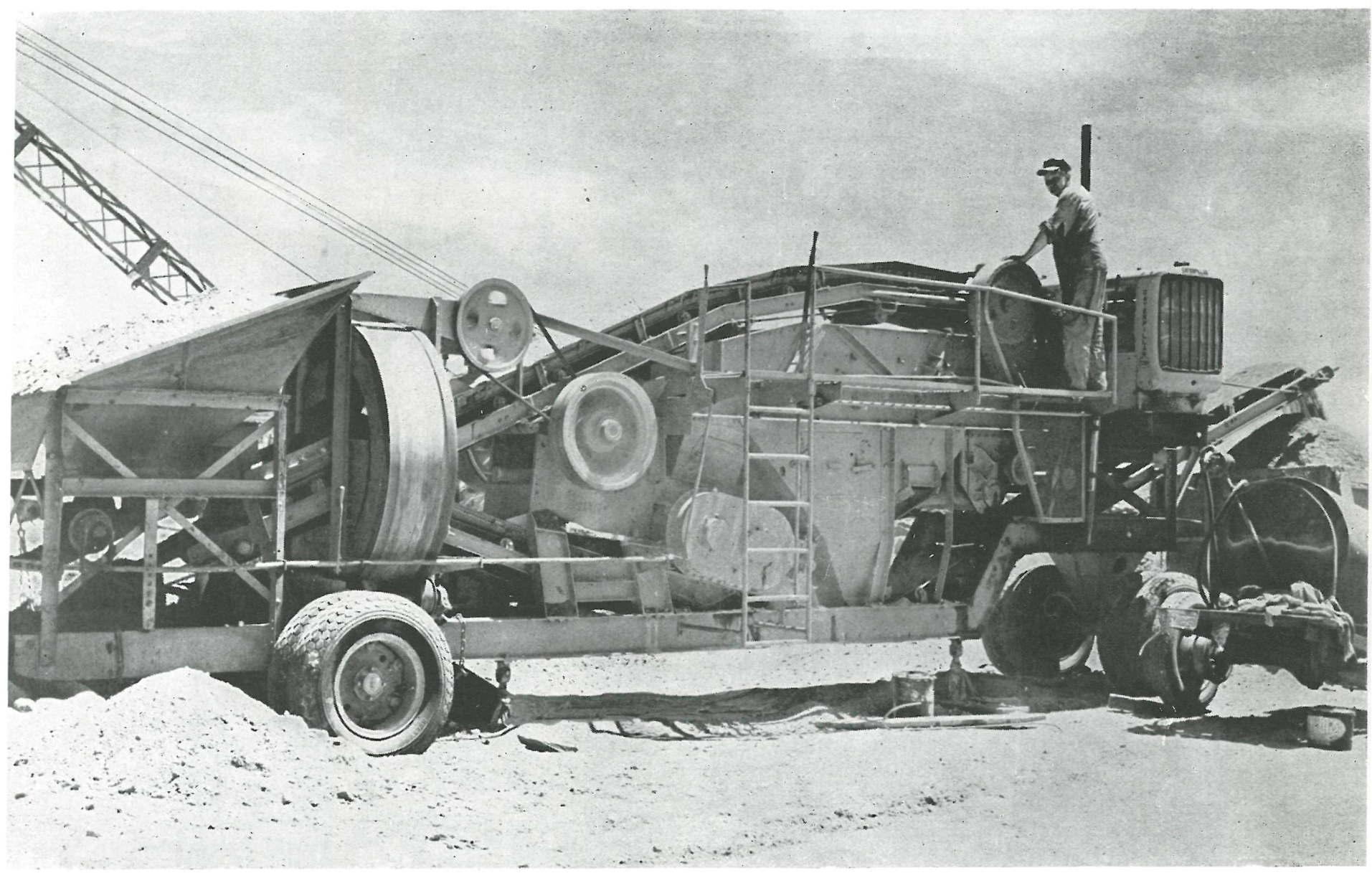

\section{Generalidades}

Para la preparación mecánica de áridos, tanto para carreteras, pistas de aeródromos, como para la construcción de estructuras de hormigón, las tendencias actuales de concentrar la producción en instalaciones móviles, caso de gran utilidad cuando la obra se extiende mucho en longitud, tienen una aplicación inmediata las centrales móviles que fabri- ca la casa americana Iowa Manufacturing International Sales Inc., de Cedarapids, de las que el Parque Central de Maquinaria del Ministerio de Obras Públicas ha adquirido diez.

Estas instalaciones disponen de medios propios para su regulación, producción y ajuste de sus órganos para concurrir en el objetivo final: regularidad, calidad y tamaños resultantes.

INSTITUTO TECNICO DE LA CONSTRUCCION Y DEL CEMENTO 


\section{central PITMASTER}

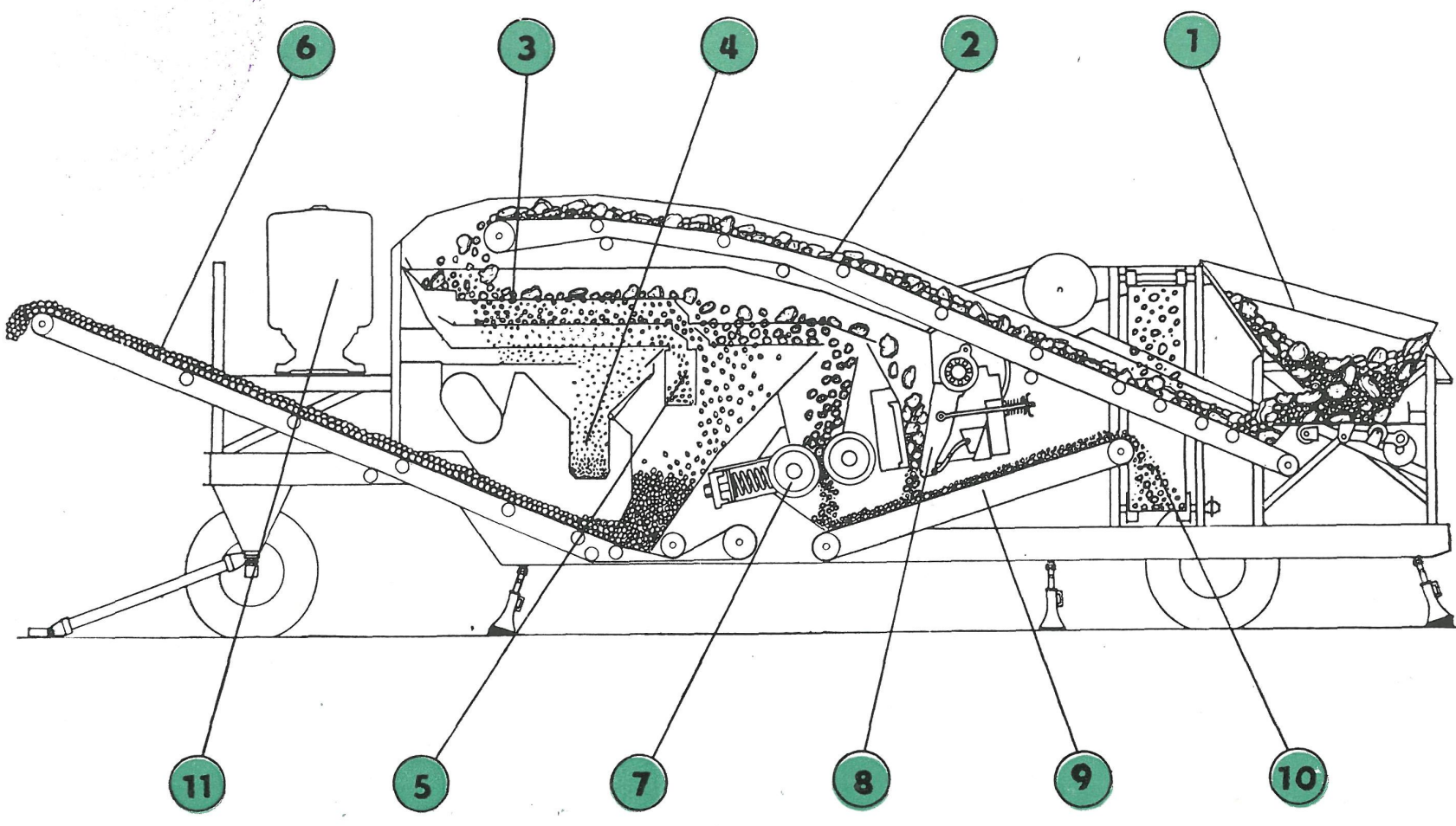

1. Tolva de carga y alimentador.-2. Transportador superior.-3. Criba vibratoria.4. Transportador (primer tamaño).-5. Transportador (segundo tamaño).-6. Transdíbulas. -9. Transportador inferior.-10. Tambor elevador de áridos.-11. Motor.

esquema de funcionamiento

Estas instalaciones pueden producir distintos tamaños de áridos, que se depositan en recipientes aislados, todo lo cual constituye una ventaja notable. La universalidad de su empleo ha sido llevada a una explotación racional en graveras y en canteras; pero, en este último caso, se necesita acoplarle un órgano primario que transforme los productos obtenidos en cantera, donde el troceo es el resultado de un juicioso uso y distribución del explosivo, por otros que se presten mejor a una alimentación directa de la central.

El esquema de funcionamieno de una central Pitmaster puede verse en la figura. Los áridos, cargados por medio de una pala mecánica o transportador de cinta en la tolva de recepción 1, en cuya parte superior suele disponerse una reja inclinada de barras, convenientemente separadas, para evitar pasen al circuito general de producción los tamaños cuyas dimensiones sean superiores a las indicadas para la machacadora de mandíbulas 8 que dispone la instalación.

Un alimentador de movimiento alternativo, cuya carrera es regulable, hace pasar los áridos de la tolva al transportador superior 2 , el cual los conduce hasta la criba vibratoria 3. Esta criba, tal como se presenta en el esquema, está dispuesta para que la central suministre áridos de tres dimensiones, de forma que al llegar a ella el material de alimentación se produce una primera clasificación, que elimina los áridos cuyas dimensiones son inferiores o iguales al tamaño máximo de piedra que se desea obtener. El transportador lateral 4, que no aparece en la figura, lleva los áridos más finos (arena) a un depósito, el 5 opera igualmente con tamaños intermedios, y el 6, frontal, con los de mayor dimensión.

El resto que queda sobre la criba, que no ha pasado por las mallas correspondientes, se clasifica en una extremidad de la criba en dos partes: una de ellas, de menor tamaño, pasa al molino de cilindros 7, y la otra, de áridos gruesos, pasa a la machacadora de mandíbulas 8. Estas dos máquinas trituran los áridos dándoles tamaños distintos en cada una de ellas, los vierten sobre el transportador inferior 9 , y éste los lleva al tambor elevador 10. Este tambor giratorio, provisto de paletas interiores convenientemente inclinadas, eleva y vierte de nuevo los áridos triturados en el transportador superior 2, que los vuelve a conducir a la criba para su clasificación.

Así, pues, se trata de un circuito cerrado, en el que, normalmente, los áridos que han pasado por el molino de cilindros 7, deben quedar clasificados y enviados al depósito después de pasar esta segunda vez por la criba; pues, de lo contrario, se crearía un aumento progresivo de materiales en el ciclo sin posibilidad de salida.

La machacadora de mandíbulas dará tamaños que se clasifican en la criba, de donde pasan a los transportadores 4, 5 y 6, y los otros pasan al molino de cilindros 7 .

Los órganos básicos de la central son:
a) Criba vibratoria 3 .
b) Molino de cilindros 4 .
c) Ma:hadora de mandíbulas 5 . 
a) Criba vibratoria.

El juego de cribas constituye el nervio de la central, se encarga de la separación directa por tamaños del material tratado y permite eliminar del ciclo los finos, lo que evita un arrastre inútil y desgaste, distribuye los materiales que han de pasar a la trituración en molino o por quijadas y, naturalmente, el rendimiento de la central depende del buen funcionamiento, disposición y dimensiones de la criba.

La disposición indicada en la flgura a permite obtener tres tamaños distintos determinados y dos transitorios que se llevan, uno al molino y el otro a la machacadora. Los movimientos de los áridos en la central se efectúan por medio de cintas transportadoras. Si llamamos, $a, b, c$, $d$, etc., a las dimensiones de estos áridos, se pueden deninir de la forma siguiente:

Arido I: comprendido entre 0 y $a \mathrm{~mm}$.

Arido II: comprendido entre $a$ y $b \mathrm{~mm}$.

Arido III: comprendido entre $b$ y $c \mathrm{~mm}$. y $d$

Aridos enviados al molino: comprendidos entre

Aridos enviados a la machacadora: mayores que $d$.

La amplitud de mallas en la criba deberá ser la siguiente:

Malla $S_{1}$, de dimensión equivalente al tamaño $c$.

Malla $\mathrm{C}_{1}$, de dimensión equivalente al tabaño $b$.

Malla $I_{1}$, de dimensión equivalente al tamaño $a$.

Malla $\mathrm{S}_{2}$, de dimensión equivalente al tamaño $d$.

Malla $I_{2}$, de dimensión equivalente al tamaño $c$.

Al definir las dimensiones de las mallas ha de tenerse en cuenta que, en la trama cuadrada, que es la única empleada por su elevado porcentaje de superficie útil de cribado, existe una diferencia entre la luz de malla y los tamaños que pueden pasar. En efecto, por una malla de un centímetro de lado pueden pasar áridos, orientados según la diagonal, de hasta $1,4 \mathrm{~cm}$ entre puntas.

La tabla núm. I da la correspondencia entre los tamaños proyectados y las luces de las mallas cuadradas correspondientes. Por ejemplo, si deseamos obtener los áridos:

Arido I: comprendido entre 0 y $1 / 2$ ".

Arido II: comprendido entre $1 / 2$ " y 1 ".

Arido III: comprendido entre 1" y 2". 3".

Aridos enviados a la machacadora: mayores de 3". Las luces de las mallas correspondientes serán:

Malla $S_{1}$, de $1 \% "$ " de luz.

Malla $C_{1}$, de $7 / 8$ " de luz.

Malla $I_{1}$, de $7 / 16$ " de luz.

Malla $S_{2}$, de $2 \frac{1}{2}$ " de luz.

Malla $I_{2}$, de $1 \%$ " de luz.

En la figura $a$ puede observarse que, modificando la posición de la compuerta X y de los paneles $\mathrm{Z}$ e $\mathrm{Y}$, pueden mezclarse, parcial o totalmente, los diferentes tamaños o cambiar la distribución en la criba para obtener dos o un solo tamaño. La figura $b$ indica la disposición para obtener un tamaño único.

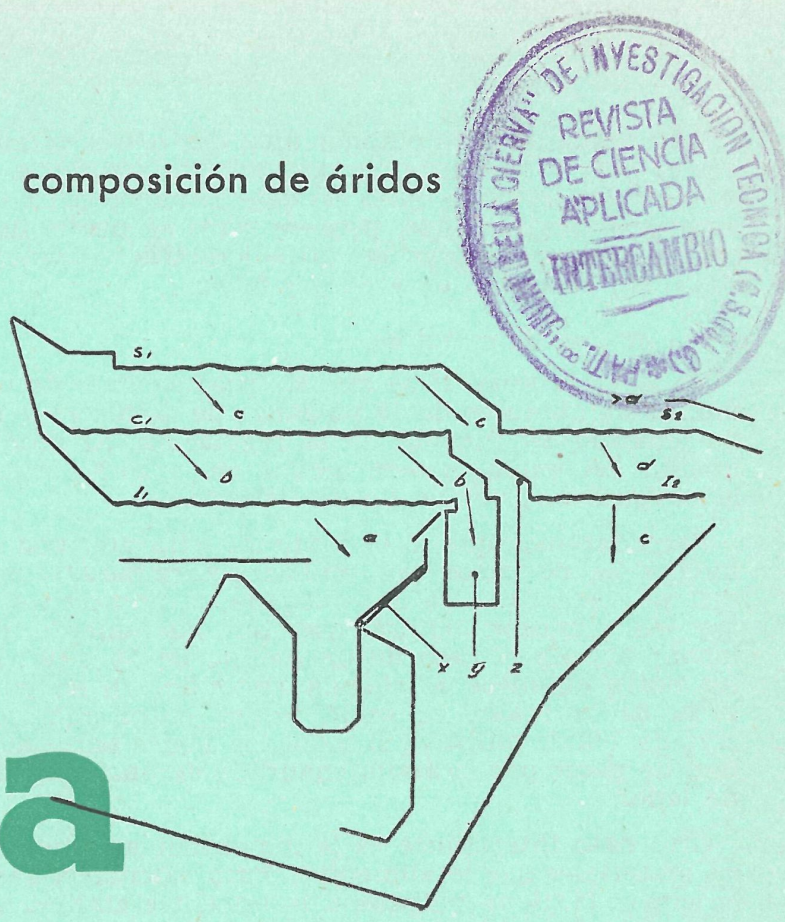

para 3 tamaños

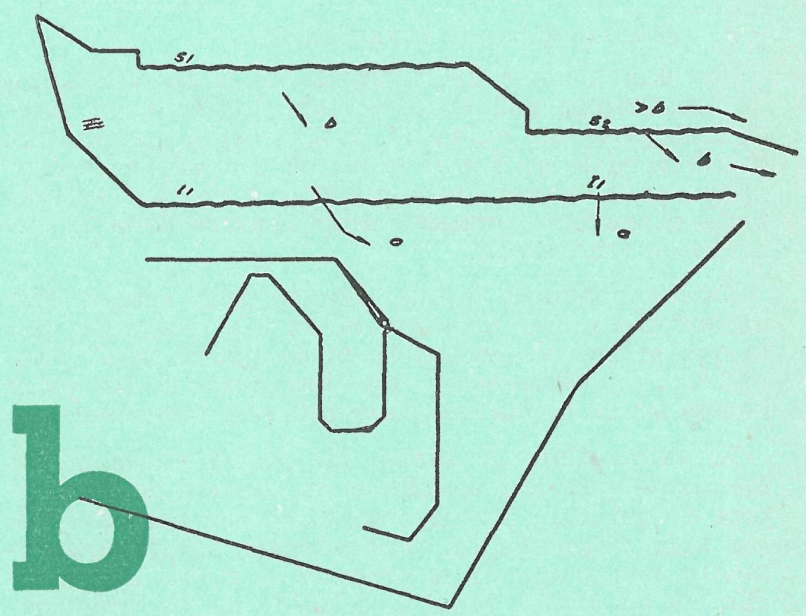

para 1 tamaño 
Fsta posibilidad de obtener uno, dos o tres tamaños es muy interesante, ya que permite adaptarse a las necesidades de la obra. En determinados casos es necesario tres tamaños, pero en otros se puede hacer mezclas de áridos de granulometría continua, con o sin exclusión de arenas y finos.

\section{b) Molino de cilindros.}

En la tabla núm. II se dan las producciones de un molino de cilindros de 16 pulgadas de diámetro y 16 de longitud en función de la regulación. El rendimiento del molino puede oscilar en $\pm 25 \%$, según la naturaleza y estado de los materiales.

Recordaremos que si el material triturado por el molino ha de pasar por mallas determinadas, por ejemplo, cuadrada de una pulgada, la abertura entre los cilindros tendrá que ser ligeramente de menor luz. En el caso de un molino de $16 \times 16^{\text {", }}$ la luz entre cilindros se calcula restando $1 / 8$ de pulgada de la malla es decir, la luz entre cilindros será de $7 / 8$ de pulgada, si todos los áridos triturados han de pasar por la malla cuadrada de una pulgada de lado.

Otro dato interesante es la dimensión máxima de los materiales que le alimentan. Para calcularla basta sumar $11 / 16$ de pulgada a la luz entre cinlindros, expresada también en pulgadas, cuando se utilicen camisas lisas de acero, ó $1 \frac{1}{8}$ pulgadas si un cilindro es liso y el otro acanalado.

Ejemplo, si la luz entre rodillos es de $1 / 2$ pulgada, la dimensión de los materiales que han de pasar será de $1 / 2 "+11 / 16^{\prime \prime}=13 / 16$ pulgadas, si ambas camisas son lisas, ó de $1 / 2+1 \%=1 \%$ pulgadas si una es lisa y la otra acanalada. Estas dimensiones máximas definen la amplitud de las mallas de la criba $S_{2}$, que da paso a los materiales que van al molino.

Las granulometrías obtenidas en el molino es función de la luz entre cilindros, es decir, de la regulación, y se indican en la tabla núm. III.

\section{c) Machacadora de mandíbulas.}

Las dimensiones del tragante de la machacadora de mandíbulas definen el tamaño máximo que puede machacar, sin embargo, la dimensión máxima del material suele ser 3/4 de la menor dimensión del tragante, para evitar atascamientos y obtener la máxima producción, manteniendo siempre llena la cámara de trituración.

La machacadora de mandíbulas de la central Pitmaster tiene una tragante de $10 \times 16$ pulgadas, por lo que la mayor dimensión de la piedra será: $10 \times$ $\times 3 / 4=71 / 2$. Dimensión que determina la luz entre barras de la rejilla colocada sobre el tragante.

La tabla núm. IV indica las producciones de las machacadoras de mandíbulas en función de la abertura de quijadas. Puede observarse que para aberturas muy pequeñas los rendimientos bajan notablemente, por lo que, en la práctica, el factor de reducción de los áridos, igual al cociente de las dimensiones de entrada y salida, no debe ser superior a 5 ó 6 , pues si es superior, el rendimiento es bajo, y, además, se produce un desgaste excesivo en las mandíbulas.

Las granulometrías que dan las machacadoras de mandíbulas dependen, como sucede en los molinos de cilindros, de la regulación, pudiéndose calcular, aproximadamente, por medio de la tabla núm. III.

\section{Cálculo de la producción}

\section{Explotación de una gravera.}

Para calcular la producción horaria aproximada de la central, una vez fijados los tamaños de áridos que se desea obtener, hemos de conocer la curva de la granulometría de la gravera, que define la cantidad de material que puede pasar directamente por la criba vibratoria sin necesidad de trituración y las cantidades que habrán de pasar por las trituradoras.

Para determinar el tipo de malla en la criba y las regulaciones óptimas, partimos de los tamaños proyectados. Un ejemplo aclarará estos puntos:

Si partimos de una curva granulométrica como la de la figura 4, y deseamos obtener los tres tipos de áridos siguientes:

\begin{tabular}{|c|c|}
\hline Arido II (gravilla) ............ & $3 / 8^{\prime \prime}-7 / 8 "$ \\
\hline Arido III (grava) & $7 / 8 "-11 / 2$ \\
\hline
\end{tabular}

Con la tabla núm. I, y partiendo de estos tamaños, determinaremos el tipo de malla cuadrada correspondiente a cada paño de la criba (fig. 3), que serán:

Malla $\mathbf{S}_{1}$ $11 / 4 "$

Malla $\mathbf{C}_{\mathbf{r}}$ $3 / 4 "$

Malla I $5 / 16^{\prime \prime}$

Malla $11 / 4 "$

Para determinar la malla correspondiente al paño $\mathrm{S}_{2}$, hemos de fijar el tamaño máximo que ha de pasar por el molino.

Regulación del molino para obtener áridos inferiores a 11/2":

$$
11 / 2 "-1 / 8^{\prime \prime}=13 \% "
$$

Tamaño máximo que pasa suponiendo un rodillo liso y otro acanalado:

$$
1 \% " s " 1 \frac{1}{s} "=21 / 2 "
$$

Malla de criba $S_{2}$ (según tabla $I$ ):

$$
2^{1 / 26 "} \approx 2 "
$$

Por tanto, del material bruto procedente de la gravera, los tamaños iguales e inferiores a 11/2" serán directamente clasificados en la criba, los áridos de tamaños comprendidos entre $1 \frac{1}{2}$ " y $2 \frac{1 / 2}{\text { " pa- }}$ sarán al molino y los superiores a $2 \frac{1}{2}$ " a la machacadora.

La regulación de esta última permitirá que los materiales triturados por la misma pasen íntegramente por el paño $S_{1}$, para evitar la recirculación en la central. De acuerdo con la tabla núm. III, la abertura de salida será de $21 / 4 "$. La tabla núm. IV da la producción de la machacadora, cuyo valor es de unas $22 \mathrm{Tn} /$ hora.

La curva granulométrica nos da los siguientes porcentajes de cada tamaño:

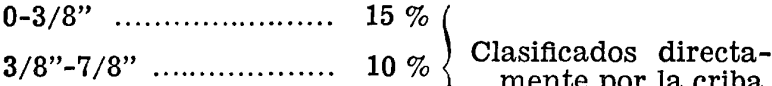

$$
\begin{aligned}
& 7 / 8 "-11 / 2 " \text { "............... } 15 \% \text { ( } \\
& 11 \frac{1}{2} "-21 / 2 " \text { "............... } 20 \% \text { Enviados al molino } \\
& \text { de cilindros. } \\
& \text { Enviados a la ma- } \\
& \text { chacadora. }
\end{aligned}
$$

Es decir, que un $40 \%$ del material tratado ha de pasar por la machacadora. Como la producción horaria admitida anteriormente es de $22 \mathrm{Tn}$, cuando la regulación es de 21/4", la cantidad total de material tratado por la planta será de $55 \mathrm{Tn} /$ hora. 


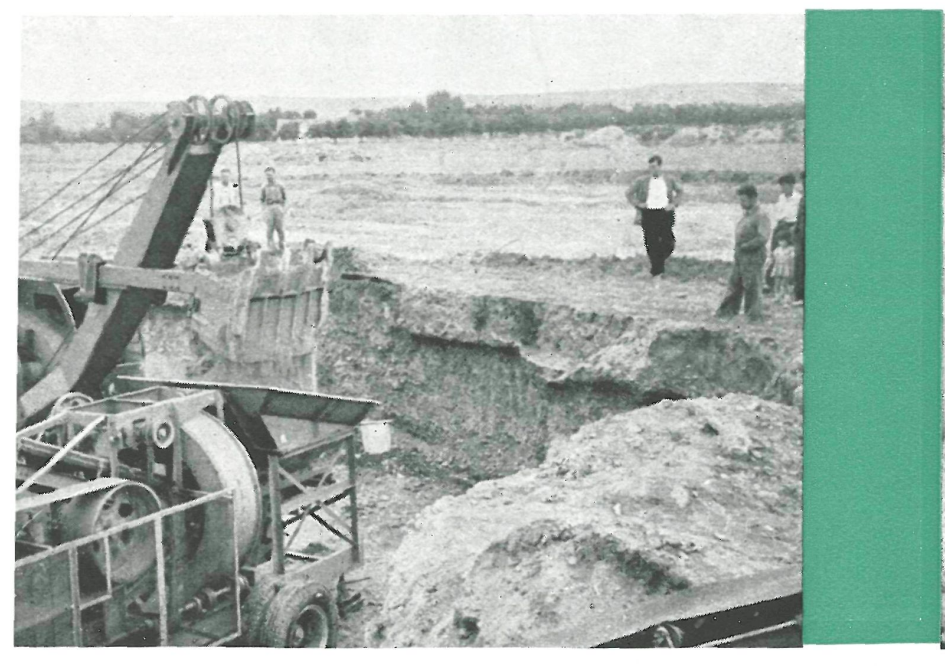

Maniobra de carga de una gravera en Alcalá de Henares.

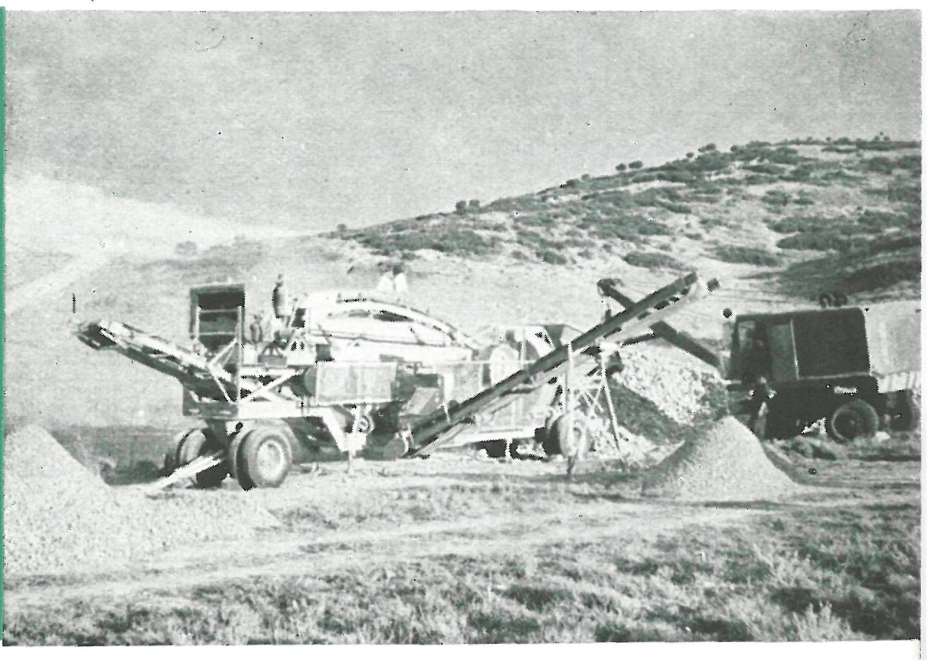

Aspecto general de la central Pitmaster instalada en la can. tera de la Marañosa.
Esta cantidad total se descompondrá, después de pasar por la criba, de acuerdo con la granulometría anterior, en las siguientes partes:

$55 \operatorname{Tn}:\left\{\begin{array}{llll}0-3 / 8, \ldots \ldots \ldots \ldots \ldots . & 15 \% & 8,25 \text { Tn clasific. } \\ 3 / 8 "-7 / 8, & \ldots \ldots \ldots \ldots & 10 \% & 5,50 \text { Tn clasific. } \\ 7 / 8 "-11 / 2 " & \ldots \ldots \ldots \ldots & 15 \% & 8,25 \text { Tn clasific. } \\ 11 / 2 "-21 / 2 " & \ldots \ldots \ldots \ldots & 20 \% & 11,00 \text { Tn molino. } \\ 21 / 2 "-7 " & \text { (máx.) } \ldots \ldots & 40 \% & 22,00 \text { Tn machacadora. }\end{array}\right.$

$$
\text { Total } \ldots \ldots \ldots \ldots .5500
$$

Según la tabla núm. III, las 22 toneladas trituradas en la machacadora nos darán la siguiente granulometría:

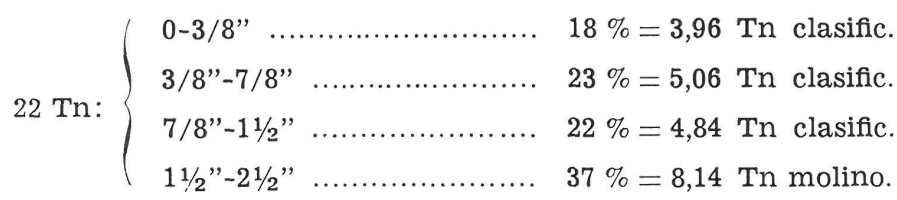

Es decir, que el molino deberá triturar, en una hora, las 11,00 toneladas de material bruto $11 / 2$ " $21 / 2$ ", más 8,14 Tn del producido de este tamaño por la machacadora, lo que da un total de 19,14 Tn. Esta cifra está muy por debajo de la capacidad de producción del molino indicada en la tabla núm. II, por lo que, en este caso, trabajará muy descansado.

La granulometría que da el molino con regulación de $1 \%, "$, obtenida en la tabla núm. III, es:

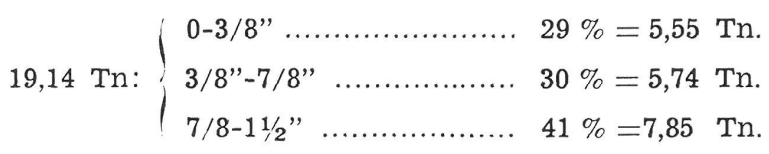

La producción total horaria se descompondrá, en tamaño, de la siguiente forma:

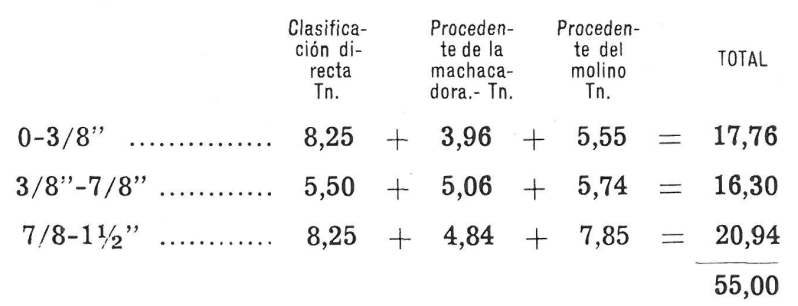

Con la disposición adoptada en el anterior ejemplo obtendremos una producción horaria de $55 \mathrm{Tn}$, de la que $32 \%$ serán tamaños $0-3 / 8$ ", $30 \%$ de $3 / 8^{\prime \prime}-7 / 8$ " y $38 \%$ de $7 / 8$ " $-11 / 2$ ".

En el ejemplo se han tenido en cuenta los decimales a fin de cuadrar la suma. Prácticamente, no se da a los cálculos tal grado de exactitud, ya que, según la naturaleza de los materiales, los porcentajes pueden variar. Los resultados calculados suelen variar de un $\pm 10 \%$ de los reales.

Observaremos que, como la capacidad de trituración del molino es superior a la de la machacadora, ésta, en la mayoría de los casos, define la capacidad total de la central en función del porcentaje de áridos contenidos en el material bruto de alimentación que ha de pasar por ella.

Basándonos en esto, hemos calculado las producciones de la central Pitmaster en función del tamaño máximo de los áridos y del porcentaje que ha de pasar por la machacadora. La tabla núm. $V$ nos da una idea de las diferencias de producción, según el tipo de gravera que se explote.

\section{Explotación de canteras.}

El arranque en cantera, aun suponiendo un troceado que pueda pasar todo el material a través de la rejilla protectora de la tolva de carga 1( fig. 1), el $100 \%$ del material de alimentación debería pasar por la machacadora. 


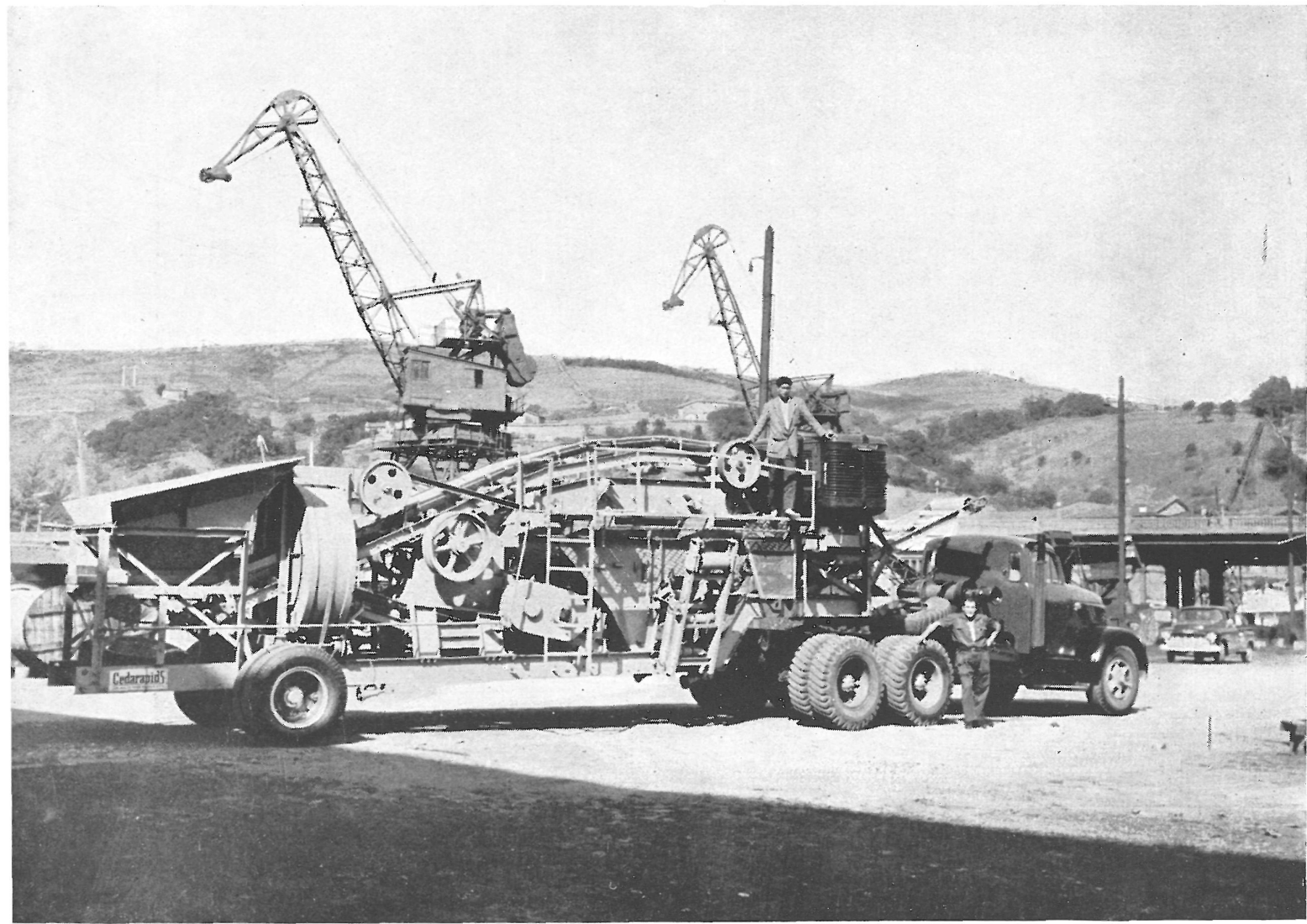

Central Pit. master, en el puerto de Bilbao, dispuesta para su transporte por ca rretera.

La producción sería baja, los paños de criba $\mathbf{S}_{1}$ y $\mathbf{S}_{2}$ experimentarían un desgaste excesivo por rozamiento, y los rendimientos de la criba y molina descenderían tanto, que la producción en la central resultaría antieconómica.

Por otra parte, como los taqueos en canteras son caros, y la tendencia actual es la de cargar directamente el material procedente del arranque sobre la machacadora, el empleo racional de una central Pitmaster en canteras necesita de un grupo primario independiente de machaqueo. Este grupo ha de ser suficientemente robusto para soportar los impactos del vaciado de las palas mecánicas sobre la machacadora, cuyas dimensiones de boca deben proporcionarse con las de la piedra de alimentación. Un transportador de cinta vierte en la tolva de la central Pitmaster los materiales que da el grupo primario.

La central trabajará como si se tratase de una gravera, en la que su granulometría se ha sustituído por lo que dara la machacadora primaria. Esta granulometría puede obtenerse en función de la abertura de mandíbulas que da la tabla núm. III.

La tabla núm. IV da la producción de las machacadoras de $15 \times 24$ y $22 \times 25$ pulgadas, indicadas para el primario de una central Pitmaster; sus rendimientos son prácticamente iguales para las regulaciones correspondientes. A primera vista puede parecer paradójica la conclusión, ya que la de $22 \times 25$ pulgadas tiene una tragante $53 \%$ mayor que la de $15 \times 24$ pulgadas, pero la relación de peso entre los bloques admitidos en una y otra están en la proporción de $22^{3} / 15^{3} \sim 3$, es decir, que la primera admite bloques tres veces más pesados y, por tanto, el trabajo de trituración es mayor.

La tabla núm. V da las producciones medias del conjunto primario-Pitmaster, según la dimensión máxima de los áridos producidos. 


\section{urva granulmétrica}

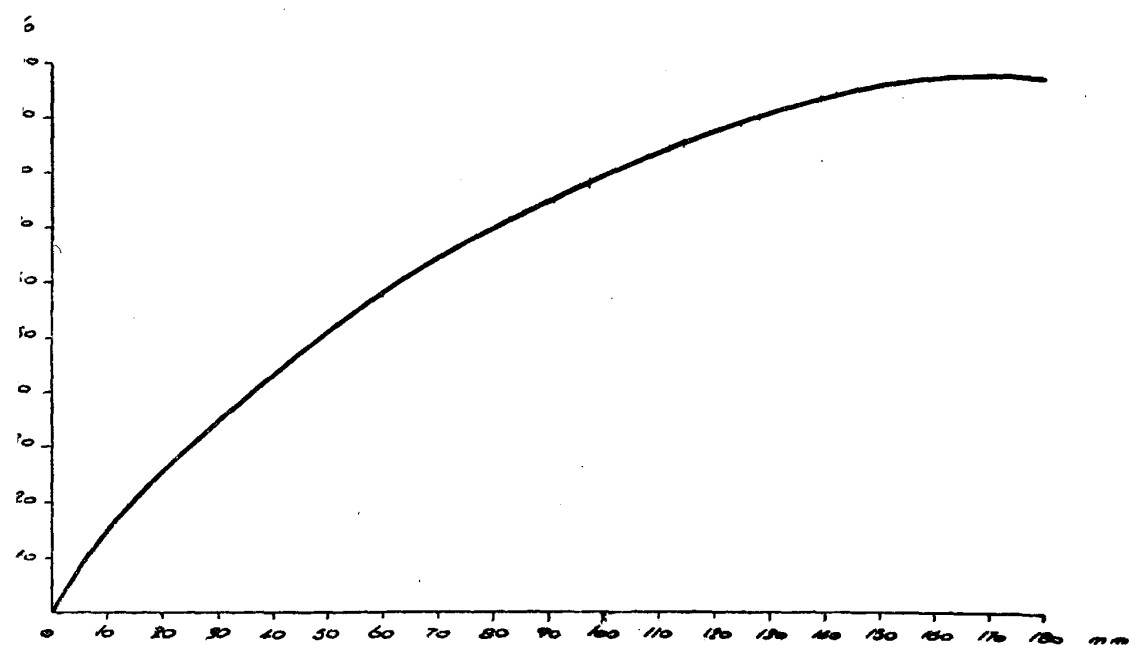

[ABLA I

Tamaños equivalentes entre mallas redondas y cuadradas de las cribas.

Las cifras indican diámetros y luces libres entre alambres.

$$
\begin{aligned}
& 1 \text { pulgada }=25,4 \mathrm{~mm} \\
& 1 \text { pulgada }=1 "
\end{aligned}
$$

\begin{tabular}{|c|c|c|c|c|c|c|c|}
\hline Redondo & $\begin{array}{l}\text { Cuadrado } \\
\text { equiv. }\end{array}$ & Cuadrado & $\begin{array}{l}\text { Redondo } \\
\text { equiv. }\end{array}$ & Redondo & $\begin{array}{l}\text { Cuadrado } \\
\text { equiv. }\end{array}$ & Cuadrado & $\begin{array}{l}\text { Redondo } \\
\text { equiv. }\end{array}$ \\
\hline $1 / 32$ & $.0257 "$ & 1/32" & $.038^{\prime}$ & $1-1 / 8 "$ & $15 / 16^{\prime \prime}$ & $1-1 / 4 "$ & $1-1 / 2$ \\
\hline $\mathbf{3} /$ & 86 & 3 & .05 & $1-1 / 4 "$ & $1 "$ & $1-3 / 8 "$ & /8 \\
\hline $1 / 16^{\prime \prime}$ & $.053 "$ & $1 / 16$ & .07 & $1-3 / 8 "$ & $1-1 / 8 "$ & $1-1 / 2 "$ & $1-3 / 4 "$ \\
\hline 3/32" & $5 / 64 "$ & 3/32" & $7 / 6$ & $1-1 / 2 "$ & $1-1 / 4 "$ & $1-3 / 4 "$ & $16 "$ \\
\hline & & $1 / 8 "$ & $5 / 3$ & $1-3 / 4 "$ & $1-1 / 2 "$ & 2" & $2-7 / 16$ \\
\hline $1 / 8 "$ & 7/64" & 6" & $7 / 32 "$ & 2" & $1-5 / 8 "$ & $2-1 / 4 "$ & $2-3 / 4 "$ \\
\hline 3/16" & 5/32" & & $5 / 16^{\prime \prime}$ & $2-1 / 4 "$ & $1-7 / 8 "$ & $2-1 / 2 "$ & 3" \\
\hline $1 / 4 "$ & 7/32" & $5 / 16^{\prime \prime}$ & $3 / 8 "$ & $2-1 / 2 "$ & $2-1 / 16^{\prime \prime}$ & 3" & $3-5 / 8 "$ \\
\hline 5/16” & $1 / 4 "$ & $3 / 8 "$ & $7 / 16 "$ & 3" & $2-1 / 2 "$ & $3-1 / 2 "$ & $4-1 / 4 "$ \\
\hline $3 / 8 "$ & 5/16" & 7/16" & $1 / 2^{\prime}$ & $3-1 / 2 "$ & $2-7 / 8 "$ & 4" & $4-3 / 4 "$ \\
\hline $7 / 16 "$ & $3 / 8 "$ & $1 / 2 "$ & $5 / 8$, & $4 "$ & $3-3 / 8 "$ & $4-1 / 2 "$ & $5-3 / 8$ \\
\hline $1 / 2 "$ & $7 / 16 "$ & $5 / 8$, & $3 / 4$ & $4-1 / 2 "$ & $3-3 / 4 "$ & 5" & 6 " \\
\hline $5 / 8 "$ & $1 / 2 "$ & $3 / 4 "$ & $7 / 8 "$ & 5" & $4-1 / 4 "$ & 6" & $7-1 / 4=$ \\
\hline $3 / 4 "$ & $5 / 8 "$ & $7 / 8 "$ & $1 "$ & 6"' & 5" & & \\
\hline $7 / 8 "$ & $3 / 4$ & $1 "$ & $1-1 / 4 "$ & $7 "$ & $5-3 / 4 "$ & 7" & $8-3 / 8 "$ \\
\hline & $7 / 8 "$ & $1-1 / 8 "$ & $1-3 / 8 "$ & 8”' & $6-3 / 4$, & 8" & $9-1 / 2$ \\
\hline
\end{tabular}

\section{TABLA II"}

Producción de un molino de cilindros, de $16 \times 16$ pulgadas, en función de la separación (regulación) entre camisas. Material de $1.600 \mathrm{~kg} / \mathrm{m}^{2}$. Para otros materiales la producción aumenta o disminuye en proporción directa al peso especiffico.

\begin{tabular}{|c|c|c|c|}
\hline Abertura & tre rodillos & Producci & an $T / h$ \\
\hline Pulgadas & Milímetros & Normales & $\begin{array}{l}\text { Desfare- } \\
\text { rables }\end{array}$ \\
\hline $1 / 4$ & 6 & 21 & 14 \\
\hline $1 / 2$ & 12 & 42 & 28 \\
\hline $3 / 4$ & 19 & 63 & 42 \\
\hline 1 & 25 & 84 & 56 \\
\hline $11 / 2$ & 38 & 124 & 83 \\
\hline $13 / 4$ & 44 & 145 & 97 \\
\hline
\end{tabular}

1 pulgada $=25,4 \mathrm{~mm}$ 


\section{TABLA III}

Porcentajes de los distintos tamaños de áridos, obtenidos con una machacador de mandíbulas, en función de la abertura de salida (regulación). Cada column vertical da los diferentes porcentajes correspondientes a cada abertura.

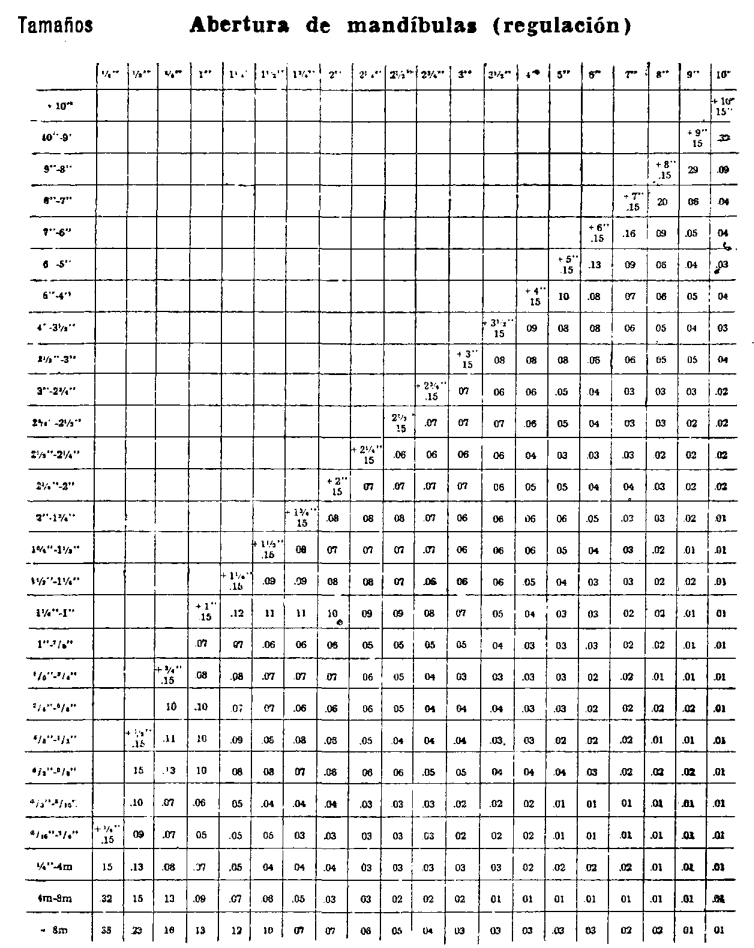

\section{TABLA IV}

Producción de machacadoras de mandíbulas en función de la abertura (regulación) entre las mismas. Material de $1.600 \mathrm{Kg} / \mathrm{m}^{3}$. La producción se halla en proporción directa al peso específico.

Machacadoras de mandíbulas

Capacidad de producción en $\mathrm{Tn} /$ hora

Peso medio del material, $1.600 \mathrm{~kg} / \mathrm{m}^{2}$

$\begin{array}{ccccc}\begin{array}{c}\text { Tamaño de la } \\ \text { machacadora } \\ \text { Abertura }\end{array} & \begin{array}{c}\text { Pulgadas } \\ \text { Milímetros }\end{array} & \begin{array}{c}\mathbf{1 0 \times 1 6} \\ \mathbf{2 5 4 \times \mathbf { 4 0 6 }}\end{array} & \begin{array}{c}\mathbf{1 5} \times \mathbf{2 4} \\ \mathbf{3 8 1} \times \mathbf{6 1 0}\end{array} & \begin{array}{c}\mathbf{2 2 \times 2 5} \\ \mathbf{5 5 9} \times \mathbf{6 3 5}\end{array} \\ \begin{array}{c}\text { Mulgados } \\ \text { Milímetros }\end{array} & 19 & & & \\ \mathbf{1}^{3 / 4} & 25 & 4-8 & - & - \\ 11 / 2 & 38 & 8-13 & - & - \\ 21 / 2 & 63 & 11-19 & 15-27 & \mathbf{2 5 - 4 5} \\ 31 / 2 & 89 & 17-28 & 25-45 & 47-75 \\ 4 & 102 & 26-42 & 40-65 & \mathbf{5 1 - 7 8} \\ \mathbf{5} & 127 & - & 48-72 & \mathbf{6 5 - 8 5} \\ \mathbf{6} & 152 & - & 65-85 & \mathbf{7 5 - 1 0 0}\end{array}$

\section{TABLA V}

Producción de una central Pitmaster en función del tamaño máximo de árido obtenido $\mathrm{y}$ del porcentaje de áridos que han de pasar por la machacadora de mandíbulas, y producciones de la misma acoplada a grupos primarios de machaques, de $15 \times 24$ y $22 \times 25$ pulgadas. Material de $1.600 \mathrm{~kg} / \mathrm{m}^{3}$.

\section{Tamaño máximo} obtenido

Pulgadas mm

$\begin{array}{cc}\text { uigadas } & \text { mm } \\ 5 / 8 & 16 \\ 7 / 8 & 22 \\ 11 / 4 & 30 \\ 1 \% / 8 & 40 \\ 2 & 50\end{array}$

Central Pitmaster

$100 \% \quad 80 \% \quad 60 \% \quad 40 \%$

$17 \quad 21 \quad 28$

23
26
$25-33$

$26 \quad 35$

$29 \quad 38$

$33 \quad 43$

\section{Pitmaster Pitmaster \\ con \\ primario primario}

$15 \times 24 \quad 22 \times 25$

$34 \quad 35$

$80 \quad 40 \quad 40$

$\begin{array}{lll}84 & 44 & 45 \\ 92 & 50 & 51\end{array}$ 